\title{
Tumor ovárico semejante a tumor de células epitelioides perivasculares (PEComa) en boga (Leporinus obtusidens)
}

\author{
Domitrovic, H.A.; González, A.O.; Flores Quintana, C.I.; Rosciani, S.A.; Hernández, D.R. \\ Instituto de Ictiología del Nordeste, Facultad de Ciencias Veterinarias, UNNE, Sargento Cabral 2139, \\ Corrientes (3400), Argentina. Tel 0379-4425753 interno 152. \\ E-mail: hdomitro@vet.unne.edu.ar
}

\begin{abstract}
Resumen
Domitrovic, H.A.; González, A.O.; Flores Quintana, C.I.; Rosciani, S.A.; Hernández, D.R.: Tumor ovárico semejante a tumor de células epitelioides perivasculares (PEComa) en boga (Leporinus obtusidens). Rev. vet. 26: 1, 63-68, 2015. Las neoplasias ováricas en teleósteos son escasamente mencionadas, existiendo más frecuencia de reportes en ejemplares de especies ornamentales. En este trabajo se describen por primera vez las características de un tumor espontáneo de ovario en un ejemplar adulto de boga (Leporinus obtusidens) obtenido del río Paraná (Argentina). Macroscópicamente, en la cavidad abdominal y en correspondencia al tercio posterior del ovario se observó una deformación irregular encapsulada de consistencia firme y aspecto quístico, con adherencias entre la membrana ovárica y el peritoneo dorsal. Muestras del tumor y de tejido ovárico fueron fijadas en formol e incluidas en parafina, obteniéndose cortes de 3 micrómetros de espesor, que se colorearon con técnicas de rutina, histoquímica e inmunohistoquímica. La observación histopatológica del tejido tumoral reveló estructuras quísticas y tejido tumoral proliferante. Las estructuras quísticas presentaron material amorfo en su interior y se encontraron delimitadas por un revestimiento celular de aspecto epitelioide. El tejido tumoral proliferante presentó un aspecto pleomórfico, hallándose zonas de tejido conectivo mixoide con células fusiformes y abundantes capilares, áreas de tejido mucoide y células poligonales cercanas a los capilares dispuestas aisladamente o formando islotes. Las células poligonales presentaron citoplasma eosinófilo y núcleos grandes con cromatina laxa y nucléolos evidentes, observándose algunas figuras mitóticas. Estas células proliferaron formando un tejido de aspecto epitelioide con capilares intercalados, adoptando un aspecto esponjoso en la periferia de algunos vasos con una importante trama de fibras reticulares. Además, se observaron delgadas fibras colágenas e infiltrado inflamatorio en la zona más externa del tejido. Las estructuras quísticas y el tejido tumoral proliferante presentaron inmunorreactividad a $\alpha$-actina. Por analogía con los tipos descriptos en mamíferos, se puede establecer que la lesión observada en ovario de boga corresponde a un tumor de origen mesenquimático, con una morfología e inmunorreactividad que se asemeja a la de los tumores de células epitelioides perivasculares (PEComa).
\end{abstract}

Palabras clave: pez Leporinus obtusidens, tumor ovárico, PEComa.

\begin{abstract}
Domitrovic, H.A.; González, A.O.; Flores Quintana, C.I.; Rosciani, S.A.; Hernández, D.R.: Ovarian tumor similar to perivascular epithelioid tumor (PEComa) in boga (Leporinus obtusidens). Rev. vet. 26: 1, 63-68, 2015. Ovarian neoplasia is scarcely mentioned in teleosts, being specimens from ornamental species the most frequently reported. This paper describes for the first time the characteristics of a spontaneous ovarian tumor from an adult specimen of boga (Leporinus obtusidens) obtained from the Paraná River (Argentina). Macroscopically, in the abdominal cavity and in correspondence to the posterior third of ovarian, an irregular firm deformation with encapsulated cystic appearance was observed, presenting adherences between the ovarian membrane and the dorsal peritoneum. Samples of tumor and ovarian tissues were fixed in formalin and embedded in paraffin. Sections of 3 micron thickness were stained with routine, histochemistry and immunohistochemistry techniques. Histopathological observation of tumoral tissue revealed cystic structures and proliferating tumoral tissue. An amorphous material was found inside the cystic structures and was defined by a cellular coating of epithelioid appearance. The proliferating tumoral tissue showed
\end{abstract}




\begin{abstract}
a pleomorphic aspect, being found areas of myxoid connective tissue with fusiform cells and abundant capillaries, mucoid tissue areas and polygonal cells placed isolated or forming islets near to the capillaries. The polygonal cells showed eosinophilic cytoplasm and large nuclei with loose chromatin and evident nucleoli, being observed some mitotic figures. These cells proliferated to form an epithelioid appearance tissue with intercalated capillaries, taking a spongy aspect on the periphery of some capillaries with a significant reticular fibers density. Also, thin collagen fibers and inflammatory infiltrate in the outermost zone of the tissue was observed. By analogy with the types described in mammals, it can be established that the lesion observed in this fish ovary corresponds to a mesenchymal origin tumor, with a morphology that resembles the perivascular epithelioid tumor (PEComa).
\end{abstract}

Key words: fish Leporinus obtusidens, ovarian neoplasia, PEComa.

\section{INTRODUCCIÓN}

En los peces, al igual que en los vertebrados superiores, las neoplasias pueden presentarse en todos los órganos y tipos de células ${ }^{26,27,29}$. Con algunas excepciones, en las cuales hay una alta prevalencia de algún tipo de tumor asociado a peces de un área geográfica específica ${ }^{14}$, la prevalencia en números globales no es alta y la importancia económica para la pesca o la piscicultura es quizá mínima ${ }^{32}$.

El estudio de las neoplasias espontáneas en los vertebrados inferiores, particularmente en peces, mostró que los tumores son morfológicamente similares y, en muchos casos, idénticos a los que ocurren en los animales superiores, incluyendo al hombre ${ }^{28}$. Además, el reporte de las neoplasias en los peces adquirió considerable importancia como un medio para detectar factores que afectan el medio ambiente, y también debido al interés biológico para entender posibles mecanismos comunes que involucran el desarrollo del cáncer en varios niveles filogenéticos, en términos de oncología comparada 25,30 .

El registro de las neoplasias espontáneas halladas en los peces fue realizado por diferentes autores ${ }^{27}$, realizándose además estudios de carcinogénesis inducida en estas especies ${ }^{4,33,34}$. El primer reporte de un tumor ovárico en peces fue de Johnstone ${ }^{19}$ que describió un cistadenoma papilar en Molva molva. Posteriormente se describió un fibrosarcoma en Gadus morhua ${ }^{36}$ y carcinomas embrionarios en Esox lucius ${ }^{12}$.

Las neoplasias ováricas en teleósteos son escasamente mencionadas como hallazgos de muestreo en especies como Cyprinus carpio ${ }^{17,31}$, Ictalurus punctatus $^{20}$, Micropterus salmoides ${ }^{24}$, Oryzias latipes ${ }^{13,15}$, Perca flavescens ${ }^{3}$, Mystus macropterus ${ }^{22}$ y en Xiphophorus maculatus ${ }^{6}$, existiendo más frecuencia de reportes en ejemplares de peces obtenidos por hibridación de especies ornamentales ${ }^{8,9,18,21}$. Otros autores realizaron estudios con marcadores antigénicos en tejidos normales y neoplásicos de peces ${ }^{5}$.

El objetivo del trabajo fue describir por primera vez las características de un tumor espontáneo de ovario con morfología similar a la de los tumores de células epitelioides perivasculares (PEComa) en un ejemplar adulto de boga (Leporinus obtusidens) obtenido del río Paraná (Argentina).

\section{MATERIAL Y MÉTODOS}

Se describen las características de un tumor espontáneo localizado en el ovario de un ejemplar de Leporinus obtusidens (longitud $36 \mathrm{~cm}$ y peso de $908 \mathrm{~g}$, Figura 1(a), obtenido del río Paraná a la altura de la localidad de Itá Ibaté (Corrientes, Argentina). En el tercio posterior del ovario, se observó una deformación irregular de 3 x 1,5 cm, encapsulada, de consistencia firme y aspecto quístico, con adherencias entre la membrana ovárica y el peritoneo dorsal (Figura 1, b-d).

El tumor y muestras de tejido ovárico se fijaron en formol $10 \%$ e incluyeron en parafina, obteniéndose cortes de 3 micrómetros de espesor que se colorearon con hematoxilina y eosina (HyE), tricrómica de Masson, impregnación de Gomory, PAS ${ }^{35}$. Para inmunohistoquímica, los cortes se colectaron en portaobjetos pre-tratados con silane (3-amino-propyltriethoxysilane; Sigma Chemical, St Louis, MO, USA), luego se desparafinaron y rehidrataron. Todas las incubaciones, excepto el anticuerpo primario, se realizaron a temperatura ambiente, en cámara húmeda, y todos los proce-

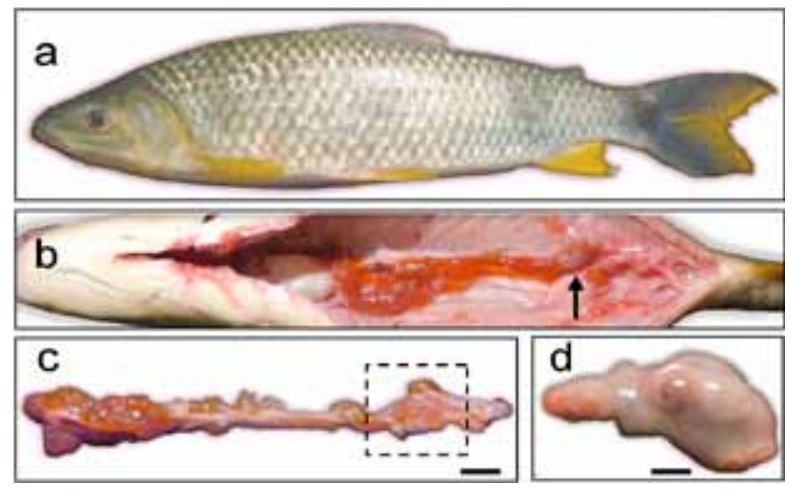

Figura 1. (a) ejemplar de L. obtusidens con tumor ovárico, (b) vista del ovario donde se observa en el tercio posterior (flecha) una deformación irregular, (c) detalle del ovario extraído de la cavidad abdominal señalando la lesión, (d) aspecto del tumor luego de su disección. Barras: $\mathrm{c}=1 \mathrm{~mm}$; $\mathrm{d}=0,5 \mathrm{~mm}$. 
dimientos de lavado consistieron en tres inmersiones sucesivas de $5^{\prime}$ en buffer fosfato salino 0,1 M (PBS; 8 $\mathrm{mM} \mathrm{N}_{\mathrm{a} 2} \mathrm{HPO}_{4}, 3 \mathrm{mM} \mathrm{N}_{\mathrm{a}} \mathrm{H}_{2} \mathrm{PO}_{4}, 150 \mathrm{mM} \mathrm{NaCl}$ ).

$\mathrm{La}$ actividad de la peroxidasa endógena fue bloqueada por incubación en solución bloqueadora $(3 \%$ $\mathrm{H}_{2} \mathrm{O}_{2}$ en PBS) por 30'. Posteriormente, las secciones fueron tratadas con leche desnatada en polvo al 3\% por $15^{\prime}$ con el objeto de bloquear reacciones cruzadas de anticuerpos no específicos. Seguidamente, las muestras fueron incubadas con $\alpha$-actina (1:50) (anticuerpo de ratón clon sm1, de laboratorios Novocastra) en cámara húmeda durante toda la noche $\mathrm{a}^{\circ} \mathrm{C}$. Finalmente, fueron incubadas con super potenciador (Super SensitiveTM Link Detection System, BioGenex, CA) en cámara húmeda por $30^{\prime}$ a temperatura ambiente, y otros $30^{\prime}$ de incubación con polímero-HRP (Super SensitiveTM Label HRP Detection System, BioGenex, CA), y más tarde reveladas con DAB (3,3 tetrahidrocloruro diaminobenzidina).

Todas las muestras se contrastaron con hematoxilina. Como controles negativos se utilizaron cortes donde el anticuerpo primario fue reemplazado por PBS. Los registros fotográficos fueron tomados con microscopio Olympus BX41 y cámara Arcano modelo 9.0.

\section{RESULTADOS Y DISCUSIÓN}

El tejido ovárico presentó una estructura histológica normal con imágenes de ovocitos correspondiente a una gónada en período de reposo (Figura 2 a). En el tejido tumoral se observaron estructuras quísticas (Figura 2) y tejido tumoral proliferante (Figuras 3 y 4). Las estructuras quísticas tenían material amorfo en su interior, delimitadas por un revestimiento celular de aspecto epitelioide (Figura 2 b-d). La cubierta celular de las estructuras quísticas estaba constituida por varias capas de células poliédricas con núcleos grandes de cromatina laxa y nucléolos pequeños, las que hacia el interior del quiste se aplanaban y necrosaban incorporando material al contenido quístico (Figura $2 \mathrm{e}$ ). Las mismas presentaron una fuerte inmunomarcación con $\alpha$-actina (Figura $2 \mathrm{f}$ ).

El tejido tumoral proliferante presentó un aspecto pleomórfico, hallándose zonas de tejido conectivo mixoide con células fusiformes y abundantes capilares, áreas de tejido mucoide y células poligonales cercanas a los capilares, dispuestas aisladamente o formando islotes (Figuras 3 y 4). Además, se observan delgadas fibras colágenas, e infiltrado inflamatorio en la zona más externa del tejido. La técnica para reticulina evidenció la presencia de una trama de fibras reticulares alrededor de estas células (Figura 3 c).

Las células poligonales poseían citoplasma eosinófilo y núcleos grandes con cromatina laxa y nucléolos bien visibles, observándose algunas figuras mitóticas (Figuras 3 b, 4 a y c). Estas células proliferaron formando un tejido de aspecto epitelioide con capilares intercalados, adoptando un aspecto esponjoso en la periferia de algunos vasos, mostrando además inclusiones citoplasmáticas eosinófilas (Figuras 3 e-f).

Las principales neoplasias reportadas en ovario de peces comprenden tumores de células tecales y granulosas, disgerminomas, teratomas, adenocarcinomas, leiomiomas, fibromas, fibrosarcomas, linfosarcomas y PEComas 1,2,11,16,21,23.

Los PEComas constituyen un grupo de neoplasias mesenquimales relacionadas que incluyen angiomiolipoma, linfangiomioma, linfangioleiomiomatosis, cap-
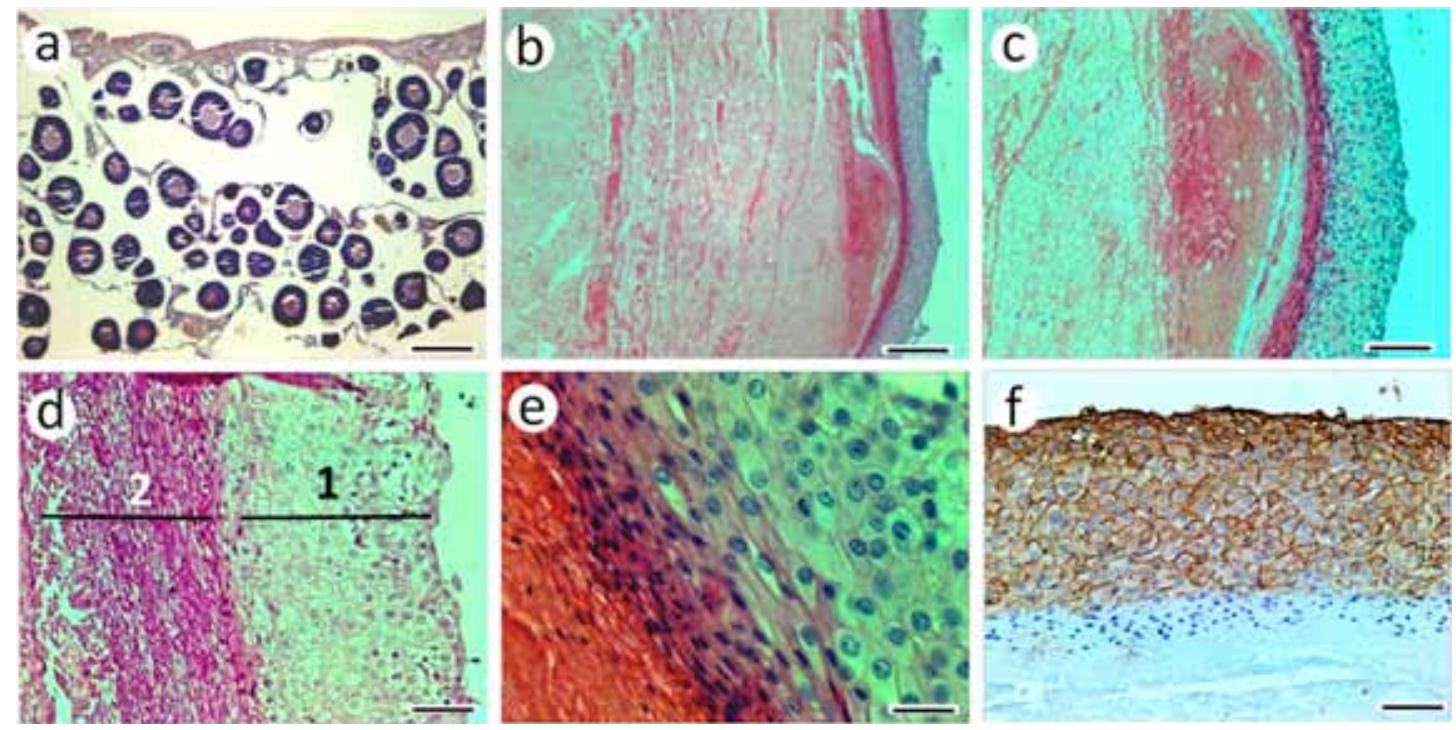

Figura 2. (a) tejido ovárico con ovocitos correspondientes al período de reposo. (b-c) aspecto de las estructuras quísticas con material amorfo y revestimiento celular de aspecto epitelioide; (d) detalle de estructuras quísticas, donde se observa el revestimiento celular de aspecto epitelioide (1) y el material amorfo hacia el interior (2); (e) se observa la cubierta celular formada por varias capas de células que hacia el interior del quiste se necrosan incorporando material al contenido quístico; (f) reacción positiva en las células de la cubierta a la inmunomarcación con $\alpha$-actina. Barras: $\mathrm{a}-\mathrm{b}=200 \mu \mathrm{m} ; \mathrm{c}=80 \mu \mathrm{m} ; \mathrm{d}$ y f $=40 \mu \mathrm{m} ; \mathrm{e}=20 \mu \mathrm{m}$. 

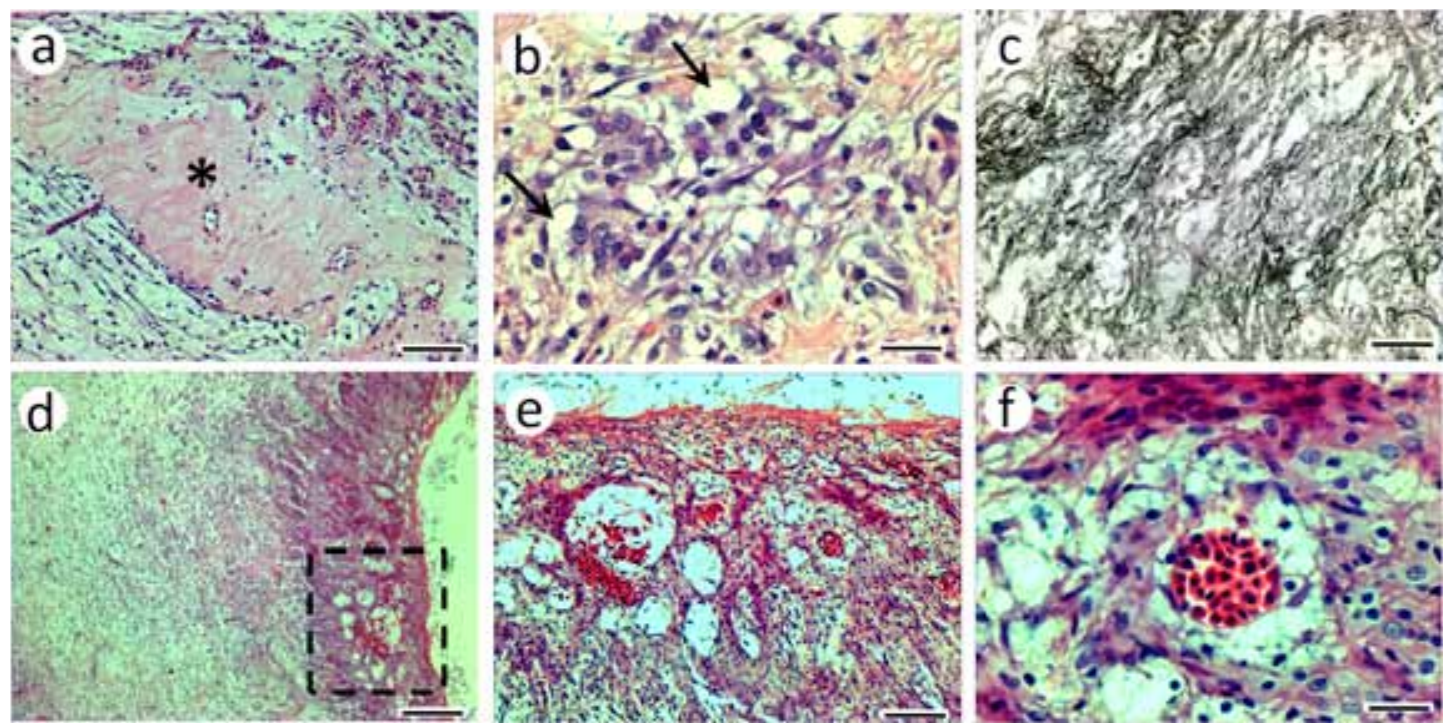

Figura 3. (a) vista del tejido tumoral proliferante con abundantes capilares y áreas de tejido mucoide (asterisco); (b) aspecto de las células poligonales, fusiformes y adipocitos (flecha); (c) detalle de fibras reticulares coloreadas con técnica de Gomory; (d) vista panorámica del tejido tumoral proliferante, (e) magnificación del recuadro de "d", (f) detalle de las células cercanas a los capilares que adoptan un aspecto esponjoso en la periferia de algunos vasos. Barras: a y e $=80 \mu \mathrm{m} ; \mathrm{b}$ y $\mathrm{f}=20 \mu \mathrm{m} ; \mathrm{c}=40 \mu \mathrm{m} ; \mathrm{d}=200 \mu \mathrm{m}$.
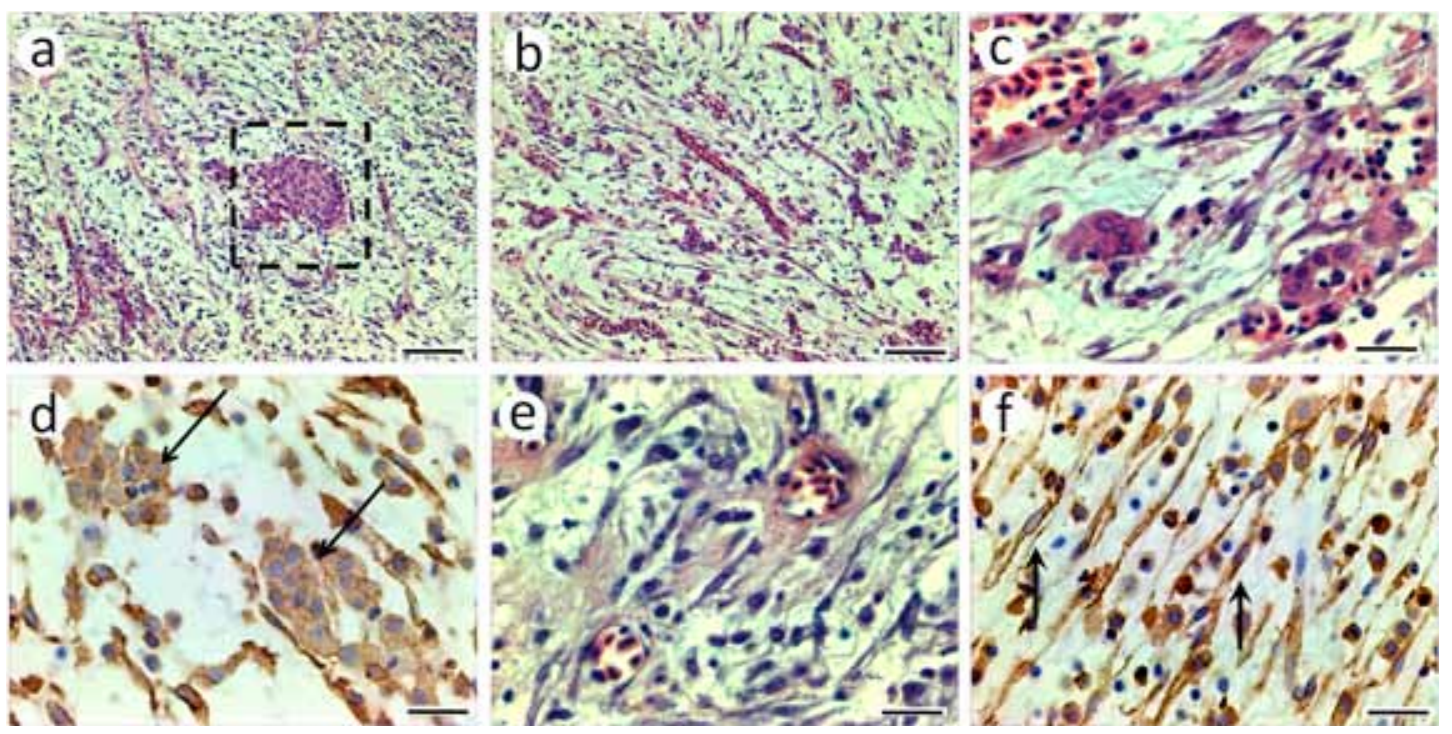

Figura 4. (a-b) área del tumor con células fusiformes, abundantes capilares y células poligonales dispuestas aisladamente o formando islotes; (c) detalle de las células poligonales; (d) reacción positiva en las células tumorales poligonales a la inmunomarcación con $\alpha$-actina (flecha); (e) detalle de células fusiformes y capilares; (f) reacción positiva en las células fusiformes a la inmunomarcación con $\alpha$-actina (flecha). Barras: $\mathrm{a}-\mathrm{b}=80 \mu \mathrm{m} ; \mathrm{c}-\mathrm{f}=20 \mu \mathrm{m}$.

suloma renal, tumor de células claras «azúcar» y tumor de células claras miomelanocitica, así como un grupo de lesiones raras que surgen en una variedad de tejidos blandos viscerales con características morfológicas e inmunohistoquímicas particulares.

Estos tumores comparten un tipo de célula distintivo, la célula epitelioide perivascular "PEC", la cual no tiene contrapartida conocida en el tejido normal. Por inmunohistoquímica, los PEComas típicamente expresan marcadores melanocíticos (HMB-45 y Melan A) y del músculo liso (actina y/o desmina), que junto a las características morfológicas permiten realizar el diag- nóstico diferencial de otros tumores, como leiomioma epitelioide y el leiomiosarcoma.

Los PEComas descriptos en seres humanos muestran un predominio femenino marcado y están compuestos de nidos y láminas usualmente epitelioides y ocasionalmente fusiformes, con citoplasma claro a granular eosinófilo y una asociación focal con paredes de los vasos sanguíneos. Se presentan más comúnmente a nivel visceral (gastrointestinal y uterino), retroperitoneal y abdominopélvicos $1,11,16,23$.

En el presente estudio se observaron estructuras quísticas y tejido proliferante con abundantes células 
epitelioides de citoplasma eosinófilo rodeando a los vasos sanguíneos, células fusiformes menos asociadas a los vasos, escasas figuras mitóticas e inmunorreactividad a $\alpha$-actina, concordando con las características mencionadas para este tipo de tumor.

La histogénesis de los PEComas es uno de los aspectos más misteriosos de la patología, proponiéndose su origen a partir de un precursor de las paredes de los vasos sanguíneos (la célula perivascular epitelioide), tal vez el pericito. Se ha propuesto que podrían tener un precursor mioblástico y también se postuló la hipótesis de su origen en la cresta neural ${ }^{10}$. El rol de los pericitos en peces teleósteos como origen de neoplasias fue analizado en Cyprinodon variegatus, sugiriéndose el uso de esta especie como modelo para el estudio de neoplasias homólogas de mamíferos ${ }^{7}$.

Por analogía con los tipos descriptos en mamíferos, se puede establecer que la lesión observada en ovario de L. obtusidens corresponde a un tumor de origen mesenquimático, con una morfología e inmunorreactividad semejante a la de los tumores de células epitelioides perivasculares (PEComa).

\section{REFERENCIAS}

1. Armah HB, Parwani AV. 2009. Perivascular epithelioid cell tumor. Arch Pathol Lab Med 133: 648-654.

2. Blazer VS. 2002. Histopathological assessment of gonadal tissue in wild fishes. Fish Physiol Biochem 26: 85-101.

3. Budd J, Schroder JD, Dukes KD. 1975. Tumors of the yellow perch. In: The Pathology of Fishes (Ribelin WE, Migaki G, Ed), University of Wisconsin Press, p. 895-906.

4. Bunton TE. 1996. Experimental chemical carcinogenesis in fish. Toxicol Pathol 24: 603-618.

5. Bunton TE, Wolfe MJ. 1996. Reactivity of tissue-specific antigens in N-methyl-N'-nitro-N-nitrosoguanidine-induced neoplasms and normal tissues from medaka (Oryzias latipes). Toxicol Pathol 24: 331-338.

6. Burns JR, Kallman KD. 1985. An ovarian regression syndrome in the platyfish, Xiphophorus maculatus. J Exp Zool 233: 301-316.

7. Couch JA. 1990. Pericyte of a teleost fish: Ultrastructure, position, and role in neoplasia as revealed by a fish model. Anat Rec 228: 7-14.

8. Dickman MD, Steele PO. 1986. Gonadal neoplasms in wild carp-goldfish hybrids from the Welland River near Niagara Falls, Canada. Hydrobiologia 134: 257-263.

9. Down NE, Leatherland JF. 1989. Histopathology of gonadal neoplasms in cyprinid fish from the lower Great Lakes of North. America. J Fish Dis 12: 415-437.

10. Fernandez-Flores A. 2011. Evidence on the neural crest origin of PEComas. Rom J Morphol Embryol 52: 7-13.

11. Folpe AL, Kwiatkowski DJ. 2010. Perivascular epithelioid cell neoplasms: pathology and pathogenesis. Human Pathology 41: 1-15.

12. Haddow A, Blake I. 1933. Neoplasms in fish: A report of six cases with a summary of the literature. J Path Bact 36 : 41-47.
13. Harada T, Okazaki N, Kubota SS, Hatanaka J, Enomoto M. 1991. Spontaneous ovarian tumour in a medaka (Oryzias latipes). J Comp Pathol 104: 187-193.

14. Harshbarger JC, Clark JB. 1990. Epizootiology of neoplasms in bony fish of North America. Sci Total Environ 94: 1-32.

15. Hawkins WE, Fournie JW, Ishikawa T, Walker WW. 1996. Germ cell neoplasms in Japanese medaka. J Aquat Anim Health 8: 120-129.

16. Hornick JL, Fletcher CD. 2006. PEComa: what do we know so far? Histopathology 48: 75-82.

17. Ishikawa T, Kuwabara N, Takayama S. 1976. Spontaneous ovarian tumors in domestic carp (Cyprinus carpio): light and electron microscopy. J Natl Cancer Inst 57: 579584.

18. Ishikawa T, Takayama S. 1977. Ovarian neoplasia in ornamental hybrid carp (Nishikigoi) in Japan. Ann N Y Acad Sci 298: 330-341.

19. Johnstone J. 1915. Diseased and abnormal conditions of marine fishes. Proc Trans Lpool Biol Soc 29: 80-113.

20. Khoo L, Camp KL, Leard AT, Harshbarger JC. 2000. Granulosa cell tumor in a farm-reared channel catfish. $J$ Aq Anim Health 12: 241-245.

21. Leatherland JF, Down NE. 2001. Tumours and related lesions of the endocrine system of bony and cartilaginous fishes. Fish Fish 2: 59-77.

22. Majeed SK, Wang DS. 1994. Tumours in the gonads of the bagrid catfish, Mystus macropterus (Bleeker). J Fish Dis 17: 527-532.

23. Martignoni G, Pea M, Reghellin D, Zamboni G, Bonetti F. 2008. PEComas: the past, the present and the future. Virchows Arch 452: 119-132.

24. Masahito P, Ishikawa T, Takayama S, Sugimura H. 1984. Gonadal neoplasms in largemouth bass, Micropterus salmoides and Japanese dace (ugui), Tribolodon hakonensis. Gann 75: 776-783.

25. Masahito P, Ishikawa T, Sugano H. 1988. Fish tumors and their importance in cancer research. $J$ Cancer Res 79: 545-555.

26. Mawdesley-Thomas LE. 1969. Neoplasia in Fish. A Bibliography. J Fish Biol 1: 187-207.

27. Mawdesley-Thomas LE. 1975. Neoplasia in Fish. In: The Pathology of Fishes (Ribelin WE, Migaki G, Ed.), University of Wisconsin Press, 805-870.

28. Meyers TR, Hendricks JD. 1983. Histopathology of four spontaneous neoplasms in three species of salmonid fishes. J Fish Dis 6: 481-499.

29. Noga EJ. 1996. Fish Diseases. Diagnosis and Treatment, Ed. Mosby Year Book, St.Louis, Missouri, USA, p. 201205.

30. Ramos P, Peleteiro MC. 2003. Three cases of spontaneous neoplasia in fish. Rev Port Cienc Vet 98: 77-80.

31. Raidal SR, Shearer PL, Stephens F, Richardson J. 2006. Surgical removal of an ovarian tumour in a koi carp (Cyprinus carpio). Aust Vet $J$ 84: 178-181.

32. Roberts RJ. 1989. Neoplasia of Teleosts. In: Fish Pathology (Roberts RJ, ed.), Bailliére Tindall, London, UK, p. 153-172. 
33. Spitsbergen JM, Tsai HW, Reddy A, Miller T, Arbogast D, Hendricks JD, Bailey GS. 2000. Neoplasia in zebrafish (Danio rerio) treated with 7,12-Diniethylbenz [a] anthracene by two exposure routes at different developmental stages. Toxicol Pathol 28: 705-715.

34. Spitsbergen JM, Tsai HW, Reddy A, Miller T, Arbogast D, Hendricks JD, Bailey GS. 2000. Neoplasia in zebrafish (Danio rerio) treated with $N$-methyl- $N$ '-nitro$N$-nitrosoguanidine by three exposure routes at different developmental stages. Toxicol Pathol 28: 716-725.
35. Suvarna KS, Layton C, Bancroft JD. 2012. Bancrofts theory and practice of histological techniques, 7th Ed., Churchill Livingstone-Elsevier, London, $654 \mathrm{p}$.

36. Thomas L. 1927. Les sarcomes fibroblastiques chez la morue. Bull Ass Fr Etude Cancer 16: 78-89. 